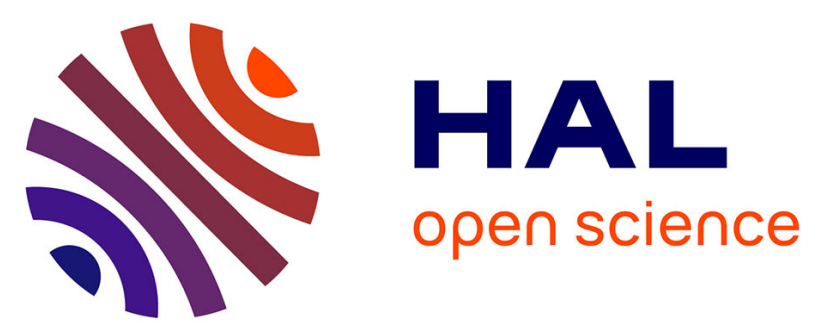

\title{
On the use of hot-wire anemometry in pulsating flows. A comment on 'A critical review on advanced velocity measurement techniques in pulsating flows'
} Arganthaël Berson, Philippe Blanc-Benon, Geneviève Comte-Bellot

\section{- To cite this version:}

Arganthaël Berson, Philippe Blanc-Benon, Geneviève Comte-Bellot. On the use of hot-wire anemometry in pulsating flows. A comment on 'A critical review on advanced velocity measurement techniques in pulsating flows'. Measurement Science and Technology, 2010, 21, pp.128001. 10.1088/09570233/21/12/128001 . hal-00566004

\section{HAL Id: hal-00566004 https://hal.science/hal-00566004}

Submitted on 8 Jun 2012

HAL is a multi-disciplinary open access archive for the deposit and dissemination of scientific research documents, whether they are published or not. The documents may come from teaching and research institutions in France or abroad, or from public or private research centers.
L'archive ouverte pluridisciplinaire HAL, est destinée au dépôt et à la diffusion de documents scientifiques de niveau recherche, publiés ou non, émanant des établissements d'enseignement et de recherche français ou étrangers, des laboratoires publics ou privés. 


\title{
COMMENT
}

\section{On the use of hot-wire anemometry in pulsating flows. A comment on 'A critical review on advanced velocity measurement techniques in pulsating flows'}

\author{
Arganthaël Berson $^{1}$, Philippe Blanc-Benon ${ }^{2}$ and Geneviève Comte-Bellot ${ }^{2}$ \\ ${ }^{1}$ Queen's-RMC Fuel Cell Research Centre, Queen's University, 945 Princess Street, Kingston, ON, \\ K7L 5L9, Canada \\ ${ }^{2}$ Laboratoire de Mécanique des Fluides et d'Acoustique, UMR CNRS 5509, École Centrale de Lyon, \\ 36 avenue Guy de Collongue, 69134 Ecully-Cedex, France \\ E-mail: arganthaelb@yahoo.com and genevieve.comte-bellot@ec-lyon.fr
}

Received 1 June 2010

Published 4 November 2010

Online at stacks.iop.org/MST/21/128001

\begin{abstract}
In their recent topical review, Nabavi and Siddiqui (Meas. Sci. Technol. 201021 042002) recommended the use of hot-wire anemometry for velocity measurements in pulsating flows, especially at high frequency. This recommendation is misleading. The procedures invoked by these authors are valid only for small-amplitude fluctuations, which are of little interest for pulsating flows. When large-amplitude velocity changes occur without flow reversal, new procedures for the correction of the nonlinearities of the hot wire are required. This case was thoroughly investigated for a constant-voltage anemometer by Berson et al (Rev. Sci. Instrum. 200980 045102). In addition, we show that when flow reversal occurs-a case most relevant to pulsating flows-accurate hot-wire velocity measurements are not possible because heat transfer is not well defined when velocity passes through zero and changes direction.
\end{abstract}

Keywords: hot-wire anemometry, pulsating flow

\section{Introduction}

In a recent topical review, Nabavi and Siddiqui proposed a 'Critical review on advanced velocity measurement techniques in pulsating flows' $[1,2]$. One of their conclusions is that 'for pulsating velocity measurements at a point, HWA [hotwire anemometry] [...] is appropriate for high frequency velocity measurements'. This statement is erroneous and the authors' recommendations are misleading. Indeed, as we shall demonstrate, the hot-wire measurement techniques described in section 3.1 of [1] are valid only under certain assumptions, which are usually not verified in pulsating flows. In the following, we will address three main types of flows: small-amplitude velocity fluctuations without flow reversal, large-amplitude fluctuations without flow reversal and finally, cases with flow reversal, which are most relevant for pulsating flows.

\section{Small-amplitude velocity fluctuations without flow reversal}

As mentioned by Nabavi and Siddiqui [1], hot-wire velocity measurements in unsteady flows need to be corrected for the thermal inertia of the wires. The energy balance of an infinitely long wire is given by equation (22) in [1], which we rewrite as

$$
\frac{m_{w} c_{w}}{\chi R_{0}} \frac{\mathrm{d} R_{w}}{\mathrm{~d} t}=I_{w}^{2} R_{w}-\left(R_{w}-R_{a}\right) f(U),
$$

where $m_{w}$ is the mass of the wire, $c_{w}$ is the specific heat of the wire material and $\chi$ is its thermal coefficient of resistance. 
$R_{0}, R_{a}$ and $R_{w}$ are the resistances of the wire at a reference temperature $T_{0}$, the temperature of the fluid $T_{a}$ and when operated in the heated mode, respectively. $I_{w}$ is the electrical current through the wire and $f(U)$ is a function of the fluid velocity $U$ that accounts for the heat flux conveyed away from the wire by the flow. Similarly, the energy balance for the corresponding ideal wire, i.e. the same wire but devoid of thermal inertia, is

$$
0=I_{w}^{* 2} R_{w}^{*}-\left(R_{w}^{*}-R_{a}\right) f(U),
$$

where the superscript * denotes ideal quantities. Combining both equations to eliminate $f(U)$ yields

$$
\frac{m_{w} c_{w}}{\chi R_{0}} \frac{1}{I_{w}^{2}} \frac{1}{R_{a}} \frac{\mathrm{d} R_{w}}{\mathrm{~d} t}+\frac{R_{w}-R_{a}}{R_{w}^{*}-R_{a}}=1,
$$

when the current is maintained constant by the anemometer $I_{w}=I_{w}^{*}$, and

$$
\frac{m_{w} c_{w}}{\chi R_{0}} \frac{1}{V_{w}^{2}} \frac{\mathrm{d} R_{w}}{\mathrm{~d} t}=\frac{1}{R_{w}}-\frac{1}{R_{w}^{*}} \frac{R_{w}-R_{a}}{R_{w}^{*}-R_{a}},
$$

when the voltage $V_{w}=R_{w} I_{w}$ across the wire is maintained constant $V_{w}=V_{w}^{*}$. We see that depending on the type of anemometer the time response of the wire to an unsteady excitation is different. The three main types of anemometers are the constant-current anemometer (CCA), the constantvoltage anemometer (CVA) and the constant-temperature anemometer (CTA). Assuming an ideal electronic circuit, which is a reasonable approximation for small fluctuations, the CTA maintains the temperature of the wire constant and, therefore, does not experience thermal inertia. Time constants for the responses of the CCA and the CVA can be deduced from equations (3) and (4), respectively [3, 4], and they are given in equation (23) of [1]. However, the derivation of the time constants relies on the major assumption that fluctuations are small with respect to the mean value of the wire resistance. This assumption is common in studies of turbulent flows, a scientific domain in which hot-wires have been used extensively for many years. Reference books include [5] for the CTA and [4] for the CCA and the CVA. Nevertheless, most pulsating flows of interest experience large-amplitude velocity fluctuations around their mean so that it is not possible to linearize equations (3) and (4) in order to derive time constants. For these cases, the procedures described in [1] are not valid.

\section{Large-amplitude velocity fluctuations without flow reversal}

In the case of large-amplitude fluctuations of the excitation term, equations (3) and (4) are subject to parametric excitation and the response of the system is nonlinear. Indeed, Corrsin [3] and Comte-Bellot and Schon [6], for the CCA, and Berson et al [7], for the CVA, demonstrated that the output of the anemometer contains higher harmonics that are not present in the excitation signal. An example of how the signal might be distorted by this effect is provided in figure 1 . Velocity fluctuations are calculated from the simulated voltage output of a CVA operating a $5 \mu \mathrm{m}$ diameter wire. Details of the simulation are described in [7]. The simulation results presented here correspond to what is referred to as 'second

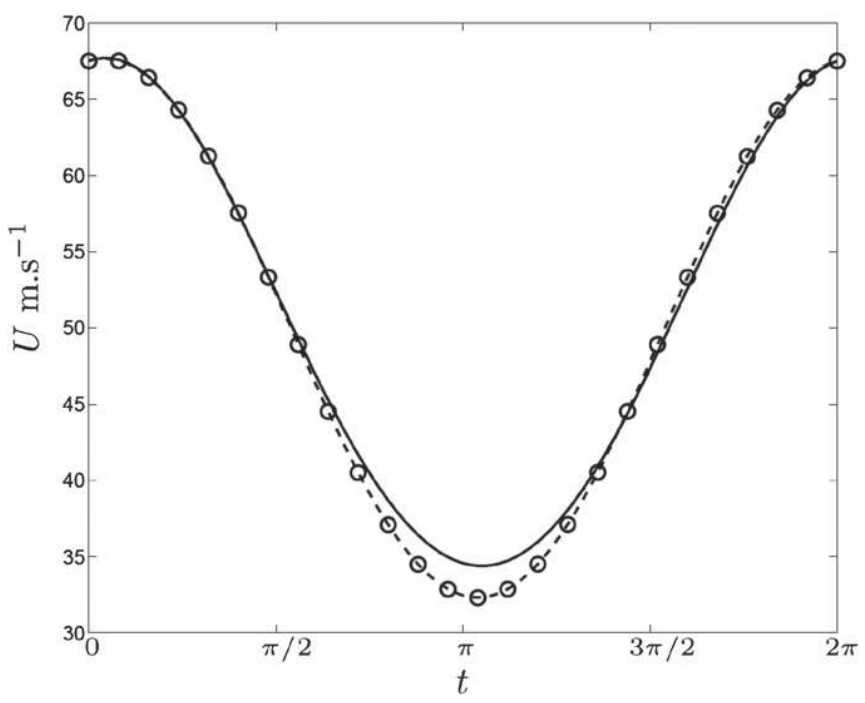

Figure 1. Time signal of velocity fluctuations over one period deduced from CVA data: correcting only for the nonlinearity of the calibration curve (full line) and after eliminating all the nonlinearities using the procedure described in [7] (circles). Simulation results are compared to the actual velocity fluctuations (dashed line). The fluid velocity is given by $U(t)=50 \times$ $\left[1+0.25 \sqrt{2} \cos \left(2 \pi f_{\text {osc }} t\right)\right] \mathrm{m} \mathrm{s}^{-1}$, where the oscillation frequency is $f_{\text {osc }}=2000 \mathrm{~Hz}$.

variant' in [7]. In that approach, the nonlinearities induced by the heat transfer-velocity relationship are corrected but not the nonlinearities induced by the thermal inertia of the wire operated in the constant-voltage mode. It is necessary to correct all the nonlinearities of the system in order to obtain accurate measurements of the pulsating flow velocity. The simple post-processing procedure described in [7] allows us to correctly recover the pulsating flow velocity for the CVA, as shown in figure 1 . The procedure relies on the numerical computation of equation (4) without any linearization. No such procedure is available for the CCA, but this type of anemometer is hardly ever used for velocity measurements nowadays.

Freymuth [8, 9] demonstrated that the CTA too has a nonlinear response. Indeed, he showed that a CTA behaves as a third-order system and that higher harmonics are generated when the amplitude of the excitation is large and the frequency is high, which distorts the output signal. In other words, it is no longer valid to assume that the resistance of a hot wire operated by a CTA is maintained constant. In addition, the CTA is usually tuned to yield an optimal bandwidth at a given mean velocity. Large departure from the operating point for which the CTA is tuned might lead to a loss in bandwidth and unstable operation. Ligęza [10] proposed a modified CTA that maintains a constant bandwidth at large velocity fluctuations and minimizes dynamic errors. However, to date, no procedure is available to correct the nonlinearities of commercial CTAs in pulsating flows.

The effect of nonlinearities on velocity measurements increases with the amplitude and the frequency of the excitation. Therefore, hot wires are not appropriate for velocity measurements in pulsating flows without flow reversal 
at high frequency, save for CVA measurements that can be adequately corrected [7].

\section{Flows with flow reversal}

Finally, we address the case of most relevance to general pulsating flows, i.e. flows with large-amplitude fluctuations and a change in the direction of the flow. First, hot wires are not sensitive to the direction of the flow. In many cases, this problem can be overcome simply by synchronizing the measurements with other available signals such as the excitation signal of the source or pressure sensors. However, the main concern is that heat transfer is not well defined near flow reversal so that no relationship between heat-transfer and the flow velocity, i.e. $f(U)$, is available. Therefore, it is not possible to measure accurately the fluid velocity in a pulsating flow with flow reversal using hot wires.

In order to illustrate our point, we perform a computation of the heat transfer between the wire and the surrounding fluid in a purely oscillating flow, i.e. with no mean velocity. We consider a wire of infinite length $\left(l_{w} / d_{w} \rightarrow \infty\right)$. The energy balance of the wire (equation (1)) is rewritten without assuming that the temperature of the wire is constant over its cross-section. The temperature $T_{w}(x, y, t)$ at a point $(x, y)$ inside the wire and a time $t$ obeys the following equation:

$$
\begin{gathered}
\frac{\partial T_{w}(x, y, t)}{\partial t}=\frac{V_{w}^{2}}{R_{0}\left(1+\chi\left(T_{w}(x, y, t)-T_{0}\right)\right)} \\
+D_{w}\left(\frac{\partial^{2} T_{w}(x, y, t)}{\partial x^{2}}+\frac{\partial^{2} T_{w}(x, y, t)}{\partial y^{2}}\right),
\end{gathered}
$$

where $D_{w}$ is the thermal diffusivity of the wire material. The source term in the wire corresponds to the Joule heating at constant voltage generated by the CVA. The local instantaneous temperature $T_{a}(x, y, t)$ of the fluid surrounding the wire is given by the energy equation of the fluid

$$
\begin{aligned}
& \frac{\partial T_{a}(x, y, t)}{\partial t}+U(x, y, t) \frac{\partial T_{a}(x, y, t)}{\partial x} \\
& =D\left(\frac{\partial^{2} T_{a}(x, y, t)}{\partial x^{2}}+\frac{\partial^{2} T_{a}(x, y, t)}{\partial y^{2}}\right),
\end{aligned}
$$

where $U(x, y, t)$ is the instantaneous velocity at point $(x, y)$ and $D$ is the thermal diffusivity of the fluid. Viscous heating is neglected. The solution of equation (6) at steady state was first derived by King [11], which led to the well-known King's law: $N u=A+B \sqrt{R e}$, where the Nusselt number $N u$ and the Reynolds number $R e$ are based on the wire diameter, and $A$ and $B$ are nearly constant. However, for an unsteady flow, no analytical solution is available for equation (6).

We use Comsol ${ }^{\mathrm{tm}} 3.3$ to solve numerically the energy equations of the wire (equation (5)) and of the fluid (equation (6)). The computational domain is depicted in figure 2. Thermal flux continuity is ensured between the wire and the fluid domains. For the sake of simplicity, we assume a potential flow where velocity fluctuations are quasistationary and the fluid is inviscid. The velocity field is given by Panton [12]. More details about the simulation can be found in [13].

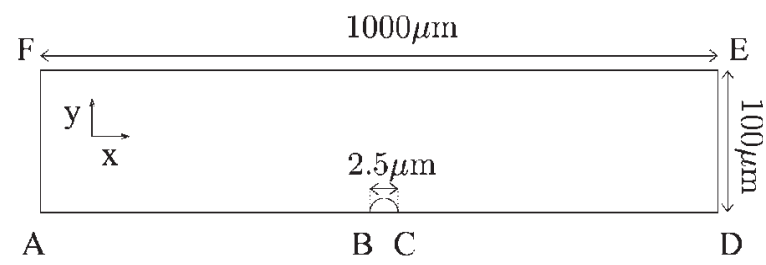

Figure 2. Computational domain for the simulation of the response of a hot wire operated by a CVA in an oscillating flow. The problem is symmetric along the axis AD. Therefore, the half disk between B and $\mathrm{C}$ represents the wire (tungsten). The rest of the domain is the fluid (air). The temperature is constant $T_{a}=300 \mathrm{~K}$ on boundaries $\mathrm{DE}, \mathrm{EF}$ and FA. Symmetry boundary conditions are imposed on $\mathrm{AB}, \mathrm{BC}$ and $\mathrm{CD}$. Thermal flux continuity is ensured at the interface between the wire and the fluid. The domain is discretized in finite elements using quadratic Lagrangian polynoms. We verified that the solutions of our computations are converged and mesh and domain independent.

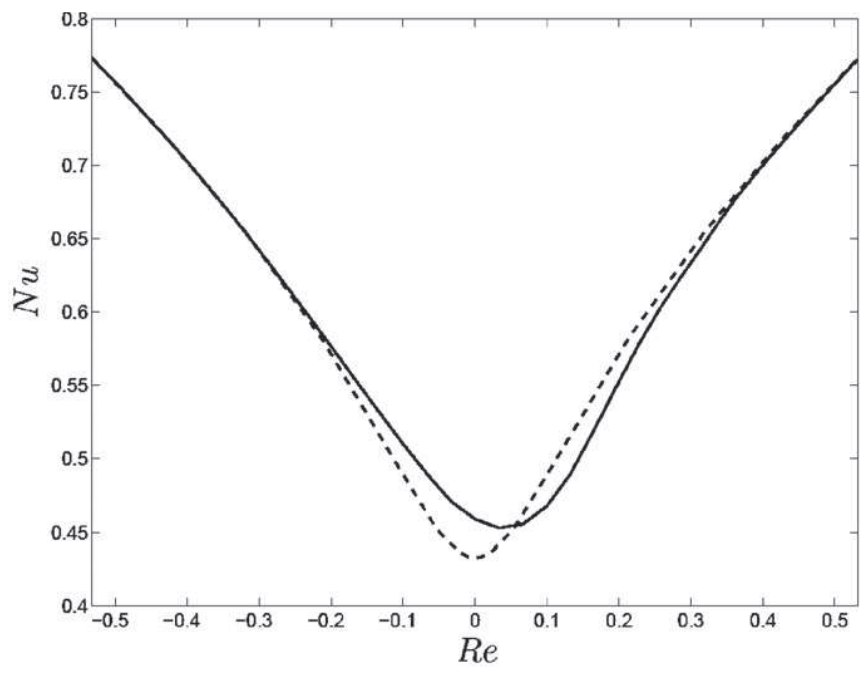

Figure 3. Evolution of the wire Nusselt number as a function of the wire Reynolds number during half a period of an oscillation. Quasi-stationary case (dashed line), simulation results (full line).

The velocity of the fluid far from the wire is a sine wave: $U(t)=5 \cos \left(2 \pi f_{\text {osc }} t\right) \mathrm{m} \mathrm{s}^{-1}$, where the frequency of oscillation is $f_{\text {osc }}=210 \mathrm{~Hz}$. Figure 3 shows the evolution of the Nusselt number integrated along the wire circumference as a function of the Reynolds number during half a period of oscillation. The half period of oscillation starts when velocity amplitude is maximum and the fluid flows towards negative $x$ coordinates $\left(U(t)=-5 \mathrm{~m} \mathrm{~s}^{-1}\right.$ or $\left.R e=-0.53\right)$. The flow changes direction at $R e=0$ and reaches a maximum of velocity amplitude, in the opposite direction, at the end of the half period $\left(U(t)=5 \mathrm{~m} \mathrm{~s}^{-1}\right.$ or $\left.R e=0.53\right)$. We compare the results obtained assuming a quasi-stationary thermal boundary layer, i.e. the results that would be obtained using King's law or similar, and the results of the unsteady simulation. Near velocity maxima, the actual Nusselt number equals the quasistationary value, i.e. heat-transfer/velocity relationships such as King's law or those obtained during calibration in a steady flow are valid. This is confirmed in figure 4(a), where the distribution of the local Nusselt number over the wire surface is exactly identical whether the flow is oscillating and reaches a 


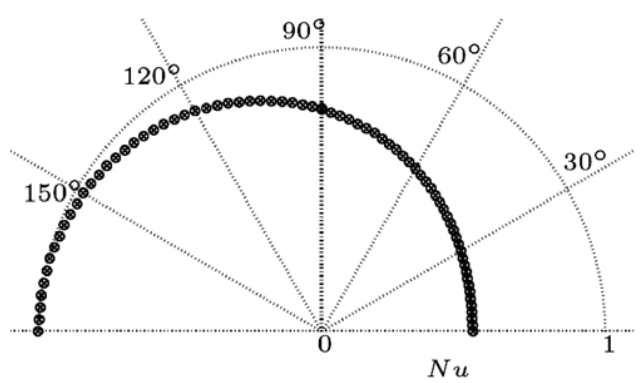

(a) $U(t)=5 \mathrm{~m} \cdot \mathrm{s}^{-1}$

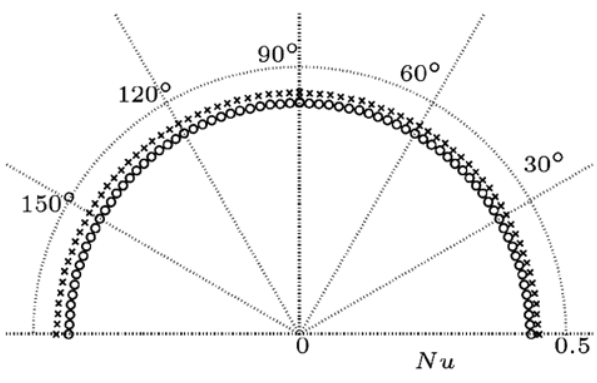

(b) $U(t)=0 \mathrm{~m} \cdot \mathrm{s}^{-1}$

Figure 4. Angular distribution of the local Nusselt number along the surface of the wire at $(a)$ a velocity maximum with the flow coming from the left, and $(b)$ at flow reversal, when the flow initially coming from the left changes direction. $\circ$ : steady flow. $\times$ : oscillating flow $U(t)=5 \cos \left(2 \pi f_{\text {osc }} t\right) \mathrm{m} \mathrm{s}^{-1}, f_{\mathrm{osc}}=210 \mathrm{~Hz}$.

velocity maximum or it is steady. However, near flow reversal, figure $4(b)$ shows that the thermal boundary layer in the oscillating flow is thinner than in the quiescent fluid, resulting in a larger local heat transfer, and it is also slightly asymmetric. Indeed, we see in figure 3 that, in the oscillating flow, the heat flux averaged over the wire is larger than predicted by the quasi-stationary approximation. In addition, heat transfer lags velocity oscillations. It is interesting to note that, near flow reversal, the same absolute Reynolds number corresponds to different Nusselt numbers, so that there is no unique correlation between fluid velocity and heat transfer.

In addition, the distortion of the signal near flow reversal becomes more important as the amplitude and the frequency of velocity oscillations increase. Such behaviour was observed previously, e.g. [14-16], and it was studied in more detail by Pedley [17-19]. We limited our computations to the thermal boundary layer, but Pedley [19] obtained similar results for the viscous boundary layer too, which affects heat transfer between the wire and the fluid even more severely. The effect is independent of the type of anemometer and prevents the accurate measurements of velocity fluctuations in pulsating flows using hot-wire anemometry.

\section{Conclusions}

In contrast to the conclusions of Nabavi and Siddiqui [1], hot-wire anemometry is not appropriate for measurements in most pulsating flows. The approach presented in [1], which concerns all types of anemometers, is limited to the case of small-amplitude velocity fluctuations with respect to the mean velocity.

When velocity fluctuations are large with respect to the mean velocity and no flow reversal occurs-a case not well addressed in [1], it is necessary to properly correct the nonlinearities of the anemometer due to the thermal inertia of the wire. The procedure proposed by Berson et al [7] corrects all these nonlinearities for the CVA. To our knowledge, no such procedure exists for the CCA or the CTA.

When velocity changes direction, there is no relationship that relates heat transfer to the flow velocity near flow reversal. Indeed, the thermal and viscous boundary layers are not the same as in a steady flow. They lag velocity oscillations and heat transfer is larger near flow reversal, which distorts the output of the anemometer. Therefore, hot-wire anemometry cannot be used to accurately measure the instantaneous velocity in pulsating flows when flow reversal occurs.

However, under certain assumptions, it is still possible to extract some valuable information from hot-wire measurements in a pulsating flow with flow reversal. For example, in a purely oscillating flow, the amplitude of velocity fluctuations at the fundamental frequency might be estimated from the maxima of the anemometer output signal [15]. Indeed, close to velocity maxima, heat transfer is similar to the quasi-stationary case and calibration curves obtained in a steady flow can be used. In addition, the fundamental frequency of the oscillations might be estimated from the period between the maxima of the anemometer output signal.

Finally, cold-wire measurements of the fluid temperature fluctuations $T_{a}$ in an oscillating flow, e.g. in an acoustic standing-wave resonator, need to be corrected for the thermal lag of the wire. The so-called time constant of the wire in fact depends on the instantaneous velocity of the surrounding fluid. An accurate procedure for the instantaneous correction of the thermal lag of the wire relies on the measurement of this 'time constant' using a hot-wire operated by a CVA [20].

\section{References}

[1] Nabavi M and Siddiqui K 2010 A critical review on advanced velocity measurement techniques in pulsating flows Meas. Sci. Technol. 21042002

[2] Nabavi M and Siddiqui K 2010 Corrigendum for 'A critical review on advanced velocity measurement techniques in pulsating flows' Meas. Sci. Technol. 21069801

[3] Corrsin S 1963 Turbulence: experimental methods Handbuch der Physik ed S Flugge vol VIII/2 (Berlin: Springer) pp 524-90

[4] Comte-Bellot G 2007 Thermal anemometry Handbook of Experimental Fluid Mechanics ed C Tropea, A L Yarin and J F Foss (Berlin: Springer) chapter B.5, sections 5.2.1-5.2.7, pp 229-83

[5] Bruun H H 1995 Hot Wire Anemometry (Oxford, UK: Oxford University Press)

[6] Comte-Bellot G and Schon J-P 1969 Harmoniques créés par excitation paramétrique dans les anémomètres à fil chaud à intensité constante Int. J. Heat Mass Transfer 12 1661-77

[7] Berson A, Blanc-Benon Ph and Comte-Bellot G 2009 A strategy to eliminate all nonlinear effects in constant-voltage hot-wire anemometry Rev. Sci. Instrum. 80045102 
[8] Freymuth P 1969 Nonlinear control theory for constant-temperature hot-wire anemometers Rev. Sci. Instrum. 40 258-62

[9] Freymuth P 1977 Further investigation of the nonlinear theory for constant-temperature hot-wire anemometers J. Phys. E: Sci. Instrum. 10 710-3

[10] Ligęza P 2007 Constant-bandwidth constant-temperature hot-wire anemometer Rev. Sci. Instrum. 78075104

[11] King L V 1914 On the convection of heat from small cylinders in a stream of fluid: determination of the convection constants of small platinum wires with applications to hot-wire anemometry Phil. Trans. R. Soc. A $214373-432$

[12] Panton R L 1996 Incompressible Flow 2nd edn (New York: Wiley) chapter 18

[13] Berson A 2007 Vers la miniaturisation des réfrigérateurs thermoacoustiques: caractérisation du transport non-linéaire de chaleur et des écoulements secondaires $P h D$ Dissertation Ecole Centrale de Lyon (in French)
[14] Elger D F and Adams R L 1989 Dynamic hot-wire anemometer calibration using an oscillating flow J. Phys. E: Sci. Instrum. 22 166-72

[15] Huelsz G and Lopez-Alquicira F 2001 Hot wire anemometry in acoustic waves Exp. Fluids $30283-5$

[16] Huelsz G, Lopez-Alquicira F and Ramos E 2002 Velocity measurements in the oscillatory boundary layer produced by acoustic waves Exp. Fluids 32 612-5

[17] Pedley T J 1975 A thermal boundary layer in a reversing flow J. Fluid Mech. 67 209-25

[18] Pedley T J 1976 Viscous boundary layers in reversing flow J. Fluid Mech. 74 59-79

[19] Pedley T J 1976 Heat transfer from a hot film in reversing shear flow J. Fluid Mech. 78 513-34

[20] Berson A, Poignand G, Blanc-Benon Ph and Comte-Bellot G 2010 Capture of instantaneous temperature in oscillating flows: use of constant-voltage anemometry to correct the thermal lag of cold wires operated by constant-current anemometry Rev. Sci. Instrum. 81015102 